\title{
Diachronie de la négation phrastique en français: apports d'une approche sociohistorique
}

\author{
BRYAN DONALDSON \\ Université de Californie, Santa Cruz \\ bryandonaldson@ucsc.edu
}

\begin{abstract}
Résumé
Cet article examine l'évolution de la négation phrastique en ancien français dans une optique sociohistorique. En ancien français, la négation simple (ne + verbe) se voit de plus en plus concurrencée par la variante novatrice renforcée $(n e+v e r b e+$ pas/mie/point $)$. Partant de l'hypothèse que la répartition des variantes conservatrice et novatrice varie en partie selon le registre, leurs fréquences sont analysées dans l'oral représenté et dans le récit, deux registres distincts au sein d'un même texte. Dans certains textes, la distribution des variantes s'avère conditionnée par le registre, l'oral représenté contenant davantage de négation renforcée que le récit. En outre, les données laissent entrevoir un effet du sexe, l'oral représenté des hommes étant plus novateur quant à la négation que celui des femmes. Ces résultats indiqueraient que le choix de forme de négation possédait une valeur sociostylistique à l'époque en question.
\end{abstract}

Mots-clés: Ancien français, négation, variation, changement diachronique

\begin{abstract}
This article examines the evolution of Old French sentential negation from a sociohistorical perspective. In Old French, simple negation (ne + verb) comes increasingly into competition with the innovative reinforced or bipartite variant $(n e+v e r b+$ pas/mie/point $)$. Starting from the hypothesis that the distribution of conservative and innovative forms varies by language register, frequencies of each form are analyzed in represented speech and in narrative, two distinct registers within a single text. In some texts, register is shown to influence the distribution of the variants, with higher rates of reinforced negation appearing in represented speech as opposed to narrative passages. Furthermore, some of the data reveal gender variation, with the represented speech of men appearing more innovative than that of women with respect to negation. The results suggest that the form of sentential negation in Old French was influenced by sociostylistic factors.
\end{abstract}

Keywords: Old French, negation, variation, diachronic change 


\section{INTRODUCTION}

L'objectif du présent article est de montrer quelques-uns des apports d'une perspective sociohistorique (Romaine 1982) sur une question pérenne dans l'histoire du français, et plus précisément en ancien français, à savoir l'évolution de la négation phrastique. Les buts d'une telle approche sont multiples : tenter d'entrevoir la situation sociolinguistique que révèlent les données historiques, retrouver, dans la mesure du possible, des traces de l'oral en ancien français, et discerner les sources et les voies de diffusion du changement.

L'évolution de la négation phrastique en français ne cesse de susciter l'intérêt des linguistes, dont la plupart y voient un exemple du cycle de Jespersen (par ex. Larrivée 2010, Mosegaard Hansen 2011, Mosegaard Hansen et Visconti 2014). Cette évolution, très familière aux diachroniciens, se déroule en gros comme en (1). (Price 1971, Foulet 1974, Harris 1988, Posner 1997, Buridant 2000, Larrivée 2011) :

(1) étape $1:$ non / ne + verbe

étape $2: n e+$ verbe + pas (mie, point etc.)

étape 3 : verbe + pas
Jeo ne dis.

'Je ne dis pas.'

Je ne dis pas.

'Je ne dis pas.'

Je dis pas.

'Je ne dis pas.'

(Larrivée 2011:1-2)

La présente étude porte un regard sociohistorique sur l'étape 2, au moment où, en ancien français et au début du moyen français, la négation simple ne se trouve en concurrence avec la négation renforcée. À l'instar de Schøsler et Völker (2014) et de Donaldson (2014), j'analyse la fréquence de la négation simple (ne) et renforcée (ne ... pas/mie) dans l'oral représenté et dans le récit, partant de l'hypothèse que la répartition de ces deux variantes (ne vs. ne...pas/mie) varie selon le registre. Un échantillon de textes de la période de 1200 à 1456 est examiné. L'hypothèse se confirme dans certains des textes examinés; par exemple, dans la Queste del Saint Graal, qui date de 1225 environ, l'oral représenté manifeste davantage de négation renforcée (64\%) que le récit (44,2\%). Qui plus est, les données provenant de l'oral représenté révèlent, du moins dans la Queste, un effet du sexe du locuteur : la grammaire des hommes dans la Queste s'avère plus novatrice que celle des femmes en ce qui concerne la négation (voir Donaldson 2014 pour des résultats semblables). Ces résultats attestent la présence de grammaires multiples en compétition, au sens de Kroch (1989), au sein d'un même texte. Les résultats soulignent également l'importance d'un étiquetage de corpus qui indique non seulement la distinction récit / oral représenté mais aussi, pour ce dernier, des renseignements de nature sociolinguistique tel le sexe du locuteur.

\section{APPROCHE SOCIOHISTORIQUE}

Partant du principe que les langues vivantes manifestent inévitablement de la variation, la sociolinguistique (Labov 1972) cherche à élucider comment et à quel point différents facteurs, y compris des facteurs sociaux (tels l'âge, le sexe ou la 
classe sociale du locuteur) et stylistiques (tel le registre) influent sur la variation et le changement linguistique. Les étapes historiques d'une langue, pour lesquelles l'accès aux locuteurs natifs n'est plus possible et pour lesquelles des enregistrements audio n'existent pas non plus, posent un problème épineux, du fait que les seules données disponibles sont celles qui proviennent de textes écrits. Comme le remarque Labov (1994), le contenu en informations sociolinguistiques des données historiques écrites est pauvre par rapport aux précisions sociolinguistiques dont on dispose pour les données ramassées sur les langues modernes. Toutefois, Romaine (1982), dans un des ouvrages fondateurs de la linguistique sociohistorique, a pu témoigner de l'intérêt d'une optique sociohistorique pour comprendre le déroulement et la diffusion des changements diachroniques, en appliquant des méthodes sociolinguistiques contemporaines à l'analyse du moyen scots. Romaine (1982) a affirmé que, pour la structure des relatives en moyen scots, différents genres de textes représentent différentes étapes d'un changement en cours; certains textes, plus conservateurs et donc assez éloignés de l'oral de l'époque, maintiennent en grand nombre les variantes conservatrices, alors que d'autres textes, telles des lettres personnelles, se rapprochent plus de l'oral et présentent en conséquence un taux plus élevé de formes novatrices. Sachant que les langues anciennes devaient présenter autant de variation que l'on observe dans les langues vivantes contemporaines, l'enjeu est d'extraire un maximum d'informations et de subtilités (sociolinguistiques et autres) des textes dont nous disposons. Autrement dit, la linguistique sociohistorique se donne comme objectif d'entrevoir la situation sociolinguistique que révèlent les données historiques écrites. C'est le procédé épousé par (entre autres) Nevalainen et Raumolin-Brunberg (2003) pour l'anglais du $15^{\mathrm{e}}$ au $18^{\mathrm{e}}$ siècle, Lodge (2004) pour le développement du français parisien, Ayres-Bennett (2004) pour le français classique, et Martineau et Mougeon (2003) pour l'omission du ne de négation dans des pièces de théâtre du $17^{\mathrm{e}}$ au $19^{\mathrm{e}}$ siècles; voir aussi Martineau (2005).

La sociolinguistique s'intéresse principalement à l'oral. Or, les diachroniciens se heurtent évidemment à une difficulté; comme le remarque Rodríguez Somolinos (2013: 7) : “On considère généralement que c'est dans l'interaction verbale qu'a lieu l'évolution de la langue et le changement linguistique. L'oral n'est cependant pas accessible pour des états de langue antérieurs." Mais les textes anciens peuvent tout de même en présenter des traces. Pour en revenir à l'axe stylistique, Koch et Oesterreicher (1985) traitent de la relation entre différents genres de texte et l'oral. En ce sens, des différences de registre dans différents textes représentent de la variation intertextuelle, un fait que Martineau $(2009,2011)$ exploite dans ses analyses de l'omission de ne et de la concurrence entre pas et point comme éléments postverbaux.

Pourtant, des différences de registre peuvent aussi exister à l'intérieur d'un seul et même texte. Par conséquent, tout comme Romaine (1982), Marchello-Nizia (2012) dissocie le récit (le narratif) et l'oral représenté (discours rapporté) - comme deux registres de langue dissemblables - au sein d'un même texte (voir aussi Fleischman 1990, Ayres-Bennett 2001). Sans nullement prétendre que l'oral représenté fournit un accès direct à la vraie langue parlée (Marchello-Nizia 2012 précise qu'il s'agit d'une représentation), ce choix méthodologique reconnaît néanmoins l'hétérogénéité stylistique que peuvent manifester les textes, surtout 
ceux d'une certaine longueur, et fait parfois ressortir de la variation intratextuelle, avec des formes grammaticales dont le taux d'occurrence varie en fonction du registre et qui correspondent à des points distincts sur la trajectoire d'un changement diachronique.

Plusieurs enquêtes sur l'ancien français ont exploité la variation intratextuelle. Price (1971) a observé que le taux d'expression du pronom sujet chez Chrétien de Troyes varie selon cet axe, avec plus de pronoms sujets exprimés dans l'oral représenté que dans le récit (voir aussi Vance 1981, cité d'après Vance 1997). De même, les résultats de Donaldson (2014; voir aussi Steiner 2014) révèlent que l'oral représenté se trouve plus en avance que le récit pour ce qui est de l'évolution vers la grammaire sujet-verbe (SV) au détriment de la grammaire plus ancienne à verbe second (V2) ${ }^{1}$. Ainsi, dans les propositions principales précédées directement d'une subordonnée, la grammaire exemplifiée par l'oral représenté se distingue de celle du récit : là où le récit manifeste des ordres de mots compatibles seulement avec la grammaire conservatrice V2, comme en (2), l'oral représenté favorise l'ordre SV, qui caractérise la nouvelle grammaire émergente, comme en (3).

(2) Ordre V2 en principale

Quant je avrai les dois d'une main ars, si ardrai je les autres.

'Quand j'aurai brûlé les doigts d'une main, je brûlerai ceux de l'autre main.'

(Roman de Cassidorus, §261; dans Vance et al. 2010:304)

(3) Ordre SV en principale

Se il nous en vuelent mener, nous irons volentiers.

'S'ils veulent nous amener, nous y irons volontiers.'

(Villehardouin 1. 181; dans Vance et al. 2010:302)

Pour Romaine (1982), ce type de disparité, dans laquelle une variante novatrice a une plus forte présence dans l'oral représenté que dans le récit, est le propre d'un changement d'en bas, diffusé à partir des couches sociales inférieures. Donaldson (2014) tire la même conclusion de ses données, qui indiquent de surcroît que l'oral représenté des hommes est plus novateur que celui des femmes dans quelques-uns des textes étudiés. Ce dernier constat trouverait son explication, selon Donaldson, dans les rôles et statuts sociaux divergents des hommes et des femmes dans la société médiévale française. Adoptant la théorie des réseaux sociaux de Milroy et Milroy (1985), Donaldson propose que les deux sexes participent généralement à des réseaux sociaux de nature différente. Quoi qu'il en soit, ces résultats témoignent de l'hétérogénéité de la grammaire - ou des grammaires en compétition (Kroch, 1989) - que peut présenter un seul texte.

\section{3. ÉVOLUTION DIACHRONIQUE DE LA NÉGATION EN FRANÇAIS}

Les chercheurs se penchent depuis longtemps sur l'évolution de l'expression de la négation en français (voir par ex. Price 1971; Foulet 1974; Harris 1988; Posner

\footnotetext{
${ }^{1} \mathrm{SV}=$ sujet-verbe $; \mathrm{V} 2$ = verbe second.
} 


\begin{tabular}{llll}
\hline \hline Étape & Variété de langue & Phrase type & Élément(s) négatif(s) \\
\hline 0 & latin classique & non dico & non preverbal \\
1 & très ancien français $9^{\mathrm{e}}$ & jeo ne dis & ne preverbal \\
& siècle -1150 environ & & \\
2 & ancien + moyen français & je ne dis (pas/miel & ne préverbal (+élément \\
& $1150-16^{\mathrm{e}}$ siècle environ & point) & postverbal) \\
3 & français classique $17^{\mathrm{e}}-18^{\mathrm{e}}$ siècle & je ne dis pas & ne +élément postverbal \\
4 & français parlé moderne $19^{\mathrm{e}}-21^{\mathrm{e}}$ & je (ne) dis pas & $($ ne $)+$ élément postverbal \\
& siècle & je dis pas & élément postverbal \\
\hline \hline
\end{tabular}

Tableau 1: Étapes de l'évolution de la négation phrastique en français

1996, 1997; Buridant 2000; Larrivée 2011) et, à en croire la place accordée au français dans deux ouvrages récents consacrés à la négation (Larrivée et Ingham 2011, Mosegaard Hansen et Visconti 2014), cette évolution ne perd en rien son attirance. Dans ses grandes lignes, elle a procédé dans l'histoire du français comme dans le tableau 1, d'après Mosegaard Hansen (2014 : 188) et Larrivée (2011: 1-2), plus élaboré que le schéma en (1).

Cette évolution, dont les grands contours semblent largement acceptés, est souvent citée comme un exemple du cycle de Jespersen (voir par ex. Larrivée 2010, Mosegaard Hansen 2011, Mosegaard Hansen et Visconti 2014).

Mais si l'évolution de la négation préverbale simple vers la négation renforcée par pas et d'autres adverbes postverbaux paraît assez claire au fil de plusieurs siècles et en prenant du recul, elle n'est pas pour autant linéaire, comme le prouve le résumé de plusieurs études précédentes établi par Schøsler et Völker (2014) et repris dans le tableau 2.

Schøsler et Völker (2014) ont également constaté que la diffusion de la forme renforcée - la variante novatrice - se fait à partir des propositions principales, pour n'atteindre que plus tardivement les subordonnées (voir aussi Mosegaard Hansen 2009a). Au cours d'un changement diachronique, une telle asymétrie n'est guère étonnante en vue du conservatisme des subordonnées par rapport aux principales (Givón 1976, Hock 1991; voir aussi Vennemann 1974). Hock (1991) cite à ce sujet l'exemple du développement de l'ordre SVO en anglais : une fois SVO devenu majoritaire dans les principales, il a fallu plusieurs siècles pour que cet ordre novateur s'étende également aux subordonnées.

En ancien et en moyen français, la négation simple en ne seul se voit de plus en plus concurrencée par la variante renforcée (avec pas, mie, etc.) et ces deux variantes coexistent dans la plupart des textes de ces périodes. Rétrospectivement, l'on sait qu'il s'agit bel et bien d'un changement diachronique et que la négation renforcée a remplacé presque entièrement la négation simple au $17^{\mathrm{e}}$ siècle. Sur le plan de la sémantique, la variante renforcée représente, à l'origine, un moyen de signaler l'emphase, de rendre plus fort le sens négatif de la phrase. Mais la présence de l'élément 


\begin{tabular}{lllc}
\hline \hline Texte & Date & $\%$ ne- $\varnothing$ & $\begin{array}{c}\text { Proportion } n e-\varnothing / \text { tous les } \\
\text { exemples de négation }\end{array}$ \\
\hline Aliscans & $1150-1200$ & $84,5 \%$ & $724 / 857$ \\
Enéas & 1160 & $76,9 \%$ & $782 / 1017$ \\
Joinville & 1309 & $68,7 \%$ & $433 / 630$ \\
Chirurgie Maître Henri & 1314 & $44,7 \%$ & $93 / 208$ \\
Bérinus & fin $14^{\mathrm{e}}$ & $73 \%$ & $1239 / 1696$ \\
XV Joies de mariage & vers 1400 & $39,5 \%$ & $17 / 43$ \\
Commynes & fin $15^{\mathrm{e}}$ & $42,6 \%$ & $182 / 427$ \\
Pathelin & fin $15^{\mathrm{e}}$ & $58,7 \%$ & $94 / 160$ \\
Jehan de Paris & fin $15^{\mathrm{e}}$ & $60 \%$ & $119 / 198$ \\
\hline \hline
\end{tabular}

Tableau 2: Déclin de la négation simple $(n e+\varnothing)$ en ancien et en moyen français (résumé de Schøsler et Völker 2014 : 148)

\begin{tabular}{|c|c|c|c|}
\hline Texte & Détails & $\begin{array}{l}\text { Date } \\
\text { approximative }\end{array}$ & $\begin{array}{l}\text { Exemples de } \\
\text { négation } \\
\text { phrastique relevés }\end{array}$ \\
\hline $\begin{array}{l}\text { Aucassin et } \\
\text { Nicolette (intégral) }\end{array}$ & $\begin{array}{l}\text { prose et vers; dialecte picard; } \\
9946 \text { mots }\end{array}$ & 1200 & 147 \\
\hline $\begin{array}{l}\text { Queste del Saint } \\
\text { Graal (extraits) }\end{array}$ & $\begin{array}{l}\text { Prose; dialecte septentrional; } \\
\text { extraits } \approx 64,000 \text { mots }\end{array}$ & 1225 & 1045 \\
\hline $\begin{array}{l}\text { Roman de } \\
\text { Cassidorus } \\
\text { (extraits) }\end{array}$ & $\begin{array}{l}\text { Prose; traits franciens; extraits } \\
\approx 54,300 \text { mots }\end{array}$ & 1267 & 1053 \\
\hline $\begin{array}{l}\text { Joinville : Vie de } \\
\text { Saint Louis } \\
\text { (intégral) }\end{array}$ & $\begin{array}{l}\text { Prose; dialecte champenois; } \approx \\
77,000 \text { mots }\end{array}$ & 1308 & 401 \\
\hline $\begin{array}{l}\text { de la Sale : Jehan de } \\
\text { Saintré (extraits) }\end{array}$ & $\begin{array}{l}\text { Prose; moyen français; extra- } \\
\text { its } \approx 175,000 \text { mots }\end{array}$ & 1456 & 745 \\
\hline
\end{tabular}

Tableau 3: Corpus et données

postverbal, suite à des processus de grammaticalisation (Posner 1997), perd la spécificité de l'emphase pour devenir peu à peu partie intégrante de la négation non marquée. Dans les textes d'ancien français, les éléments postverbaux comme pas "avaient déjà perdu en partie leur force expressive" (Wartburg 1971: 108) pour "devenir le mot normal" peu de temps après (p. 133). Einhorn (1974) considère qu'en ancien français, l'ajout d'un élément postverbal ne contribue que peu de sens, et Guiraud (1964) met en doute l'idée que la négation en ne...pas/mie renforce le sens négatif de la phrase. Pour Guiraud, si la variante renforcée comportait 


\begin{tabular}{ll}
\hline \hline Texte & Élément négatif postverbal \\
\hline Aucassin & mie, pas \\
Queste & goute, mie, pas \\
Cassidorus & goute, mie, pas \\
Joinville & mie, pas \\
Saintré & mie, pas, point (adverbial) \\
\hline \hline
\end{tabular}

Tableau 4: Inventaire d'éléments négatifs postverbaux analysés

\begin{tabular}{lcc}
\hline \hline Texte & Type ne-pas / types ne+ne-pas & \% négation renforcée \\
\hline Aucassin & $32 / 55$ & $58,2 \%$ \\
Queste & $169 / 294$ & $57,5 \%$ \\
Cassidorus & $118 / 369$ & $32,0 \%$ \\
Joinville & $43 / 76$ & $56,6 \%$ \\
Saintré & $78 / 220$ & $35,5 \%$ \\
\hline \hline
\end{tabular}

Tableau 5: Négation simple et négation renforcée en déclarative principale

vraiment un sens plus fort que ne seul, l'on s'attendrait à en voir autant en subordonnée qu'en principale, ce qui est loin d'être le cas (comme le montreront les données dans les tableaux 5 et 6). On peut en conclure que les deux variantes (ne seul et ne...pas/mie/point) possèdent déjà en ancien français une fonction identique ou presque, à savoir de marquer la négation tout court. Comme le remarque Marchello-Nizia (2003: 63), "En ancien français, les deux possibilités, morphème unique ne et morphème double ne...pas / mie, alternent sans qu'il soit possible d'y voir une signification différente."

Un constat qui ressort des chiffres du tableau 2, c'est que la négation simple est restée en concurrence avec la négation renforcée pendant une assez longue période (voir aussi Martineau 2009: 171, Mosegaard Hansen 2009a: 166). Selon Kroch (1989), il s'agit dans une telle situation de deux variantes grammaticales, et par conséquent deux grammaires, en compétition. Pendant la durée du changement, les locuteurs manifestent une sorte de diglossie, ils sont capables de se servir tantôt de la grammaire qui produit la variante conservatrice (ne), tantôt de la grammaire qui produit la variante novatrice (ne...pas).

Cependant, la coexistence de plusieurs variantes, surtout sur une longue période, se révèle propice à la différenciation des variantes sur des axes autres que la sémantique, par exemple celui du registre ou de la pragmatique (Martineau et Mougeon 2003, Nevalainen et Raumolin-Brunberg 2003). Plusieurs enquêtes récentes ont tenté de mettre au jour des significations, au-delà du sémantisme négatif de base, qui pourraient éclairer en partie le choix entre les variantes simple et renforcée au cours de ce changement, qui s'étale sur au moins cinq siècles. 


\begin{tabular}{lcc}
\hline \hline Texte & Type $n e-p a s /$ types $n e+$ ne-pas & $\%$ négation renforcée \\
\hline Aucassin & $2 / 36$ & $5,6 \%$ \\
Queste & $71 / 348$ & $20,4 \%$ \\
Cassidorus & $36 / 331$ & $10,9 \%$ \\
Joinville & $29 / 168$ & $17,3 \%$ \\
Saintré & $53 / 264$ & $20,1 \%$ \\
\hline \hline
\end{tabular}

Tableau 6: Négation simple et négation renforcée en subordonnée

Ainsi Mosegaard Hansen (2009b) et Mosegaard Hansen et Visconti (2009) émettentelles l'hypothèse que le choix entre ne et ne...pas dépend de la structure informationnelle du contenu de la proposition. Plus précisément, la négation renforcée en ne-pas (mie, etc.) s'emploie quand l'information dans la proposition est déjà connue, alors que la négation en ne seul correspond en général à l'introduction d'un nouveau référent ou d'une information inconnue. Par exemple, chez Joinville (1308 environ), Mosegaard Hansen et Visconti découvrent, dans des propositions négatives contenant un nouveau référent discursif, un taux de ne simple de $78 \%$. En revanche, les propositions contenant un référent discursif déjà connu (déjà mentionné auparavant dans le discours) favorisent la négation renforcée. Le choix de variantes négatives dans ces données serait donc, au moins en partie, liée à la structure informationnelle. Pourtant, cette hypothèse ne fait pas l'unanimité; elle est mise en cause, par exemple, par les résultats de Larrivée (2010). Dans un corpus de lettres anglo-normandes, Larrivée ne trouve pas une telle répartition de variantes selon le statut informationnel des référents. De même, l'hypothèse de la structure informationnelle n'est pas soutenue par Schøsler et Völker (2014).

D'autres enquêtes ont cherché à entrevoir une signification sociolinguistique dans les variantes négatives. C'est l'approche de Völker (2007), qui examine la concurrence entre deux formes renforcées, ne...pas et ne...mie, dans un corpus de chartes. Völker voit une association entre ne...pas et le langage de la chancellerie, dont l'influence sociale aurait contribué à la diffusion de ne...pas au détriment de ne...mie.

L'attention portée sur les apports sociolinguistiques potentiels de l'oral représenté remonte au moins à Price (1962). Chez Adam le Bossu, auteur d'expression picarde, Price décèle dans l'emploi de ne...pas une influence parisienne, car les personnages ayant un lien à Paris emploient davantage de ne...pas, alors que les autres emploient plutôt ne...mie. Price exploite la distinction entre oral représenté et récit pour appuyer ce qu'il considère comme un autre exemple de contact linguistique : chez le chroniqueur wallon Froissart, ne...pas s'avère plus fréquent que ne... mie dans l'oral représenté, alors que le récit manifeste la situation inverse. Price voit encore l'influence parisienne dans la grammaire de l'oral représenté. Pour ce qui est du choix entre la négation simple et renforcée, Glikman et Mazziotta (2014) rapportent une association (mais pas très forte) entre la négation renforcée et l'oral représenté dans la Queste del Saint Graal. En revanche, Schøsler et Völker (2014) ne trouvent pas de différences de taux de négation simple et renforcée en comparant 
les passages narratifs et l'oral représenté du Charroi de Nîmes, contrairement à leurs attentes.

\section{4. ÉTUde}

La présente étude se concentre sur la concurrence entre la négation simple et la négation avec renforcement postverbal (mie, pas, [point adverbial]) pendant l'étape 2 de l'évolution décrite dans le tableau 1. L'approche est celle de la linguistique sociohistorique et vise à dévoiler des significations sociostylistiques associées aux formes de négation en variation. Au niveau global, l'objectif est d'enrichir notre connaissance de la diffusion de la négation renforcée en français. Plus particulièrement, je mettrai à l'épreuve l'hypothèse que, lors d'un changement diachronique, la distribution des variantes est en partie déterminée par le registre. Ici, il s'agit de l'opposition entre oral représenté et récit dans un même texte. Je chercherai aussi, dans les exemples d'oral représenté, un effet du sexe du "locuteur" (suivant Donaldson 2014). Enfin, compte tenu de l'observation que les innovations linguistiques se manifestent d'abord en principale avant d'atteindre les subordonnées, ces deux types de propositions seront examinés séparément.

Quoique la distinction entre oral représenté (ou discours direct) et récit soit étiquetée dans des corpus telle la Base de Français Médiéval (BFM 2012) ou le corpus MCVF (Modéliser le changement : les Voies du français; Martineau et al. 2010), le sexe du locuteur ne l'est pas. Pour cette raison j'ai choisi de constituer manuellement un corpus de plusieurs textes datant d'entre 1200 et 1456 (tableau 3); à l'exception des passages en vers d'Aucassin et Nicolette, il s'agit de textes en prose.

Dans chaque texte ou extrait de texte, tous les exemples de négation phrastique susceptibles de varier entre la forme simple et la forme remplacée ont été relevés, soit au total 3391 exemples. Dans le cas des négations avec ne suivi d'aucun, jamais, plus et ainsi de suite, l'élément postverbal apporte au sémantisme négatif de base une valeur sémantique additionnelle. Il s'ensuit que ces négations ne manifestent pas d'alternance entre la forme simple en ne et la forme double; pour cette raison, elles n'entrent pas dans l'analyse (voir aussi Price 1997). Les données excluent également le ne de coordination ainsi que le ne explétif, dont ni l'un ni l'autre ne marque la négation phrastique.

\section{RéSultats}

Comme élément postverbal, pas se trouve en concurrence dans tous ces textes avec mie et parfois avec point (adverbial) ou goute. Le tableau 4 dresse l'inventaire des éléments négatifs postverbaux attestés. Quel que soit l'élément postverbal, ces différents cas de figure sont tous analysés désormais en tant que négation renforcée (par opposition à la négation simple). Même s'il serait souhaitable d'examiner le comportement individuel de pas, mie, goute et point adverbial, le choix de les amalgamer se justifie pour plusieurs raisons : (a) la présente étude examine le choix global entre la négation simple et renforcée; (b) l'alternance entre pas et 
mie est le plus souvent d'ordre dialectal plutôt que sémantique (Price 1997); (c) certaines formes sont très faiblement attestées dans certains textes (un seul exemple de pas dans Aucassin, relativement peu de mie dans Cassidorus); (d) mie est de moins en moins attesté, plus on s'approche du moyen français (O'Connor 1881); (e) ces éléments font tous partie de la même classe selon Price (1997), à savoir celle des particules négatives au sens strict, qui donnent une polarité négative à la totalité de la phrase (à la différence d'éléments comme personne ou nul qui n'introduisent qu'une négation partielle).

Alors que l'évolution diachronique en faveur de la négation renforcée est une évidence en comparant par exemple l'ancien français au français classique, elle demeure assez peu visible dans cet échantillon de textes. En proposition principale, contrairement à la situation à laquelle on pourrait s'attendre, c'est le plus ancien texte (Aucassin) qui présente le taux le plus élevé de négation renforcée, vraisemblablement la variante novatrice (tableau 5); les chiffres de la Queste et de Joinville n'en diffèrent que de peu, alors que Cassidorus et Saintré contiennent sensiblement moins de négation renforcée.

En subordonnée, par contraste, l'on observe une tendance croissante pour la forme renforcée, sans pour autant que la progression soit nettement linéaire (tableau 6). L'asymétrie entre principales et subordonnées observée par Schøsler et Völker (2014) se reproduit dans les présentes données, car, pour n'importe quel texte du corpus, la négation renforcée reste toujours plus fréquente en principale qu'en subordonnée. La différence est parfois frappante, par exemple dans la Queste (20,4 \% de négation renforcée en subordonnée, 57,5 \% en principale). Pour l'ensemble du corpus, cette différence de distribution des variantes simple et renforcée est statistiquement significative, $t=5,08923, p<.001$.

Dans Aucassin et Nicolette, il n'y a aucun effet significatif de registre sur la distribution des variantes négatives simple et renforcée, ni en principale, $\chi^{2}(2, N=4)=$ $0,85, p=.357$, ni en subordonnée, test exact de Fisher, $p=.124$. Le tableau 7 présente la distribution des variantes selon le registre en principale; le tableau 8 présente celle dans les subordonnées. Dans ce texte, la négation renforcée n'est que faiblement représentée en subordonnée ( 2 exemples seulement $)^{2}$.

Aucassin et Nicolette pose un défi à l'analyse de l'effet du sexe des locuteurs : à part Nicolette, ce texte ne comporte pas de personnages féminins importants, et l'oral représenté de Nicolette ne compte que neuf exemples de négation phrastique, répartis très inégalement entre les principales (huit exemples, tableau 9) et les subordonnées (un seul exemple).

Malgré l'insuffisance des données, les chiffres (pour les principales) dans le tableau 9 donnent à penser que la grammaire de la négation que manifeste

\footnotetext{
${ }^{2}$ Aucassin et Nicolette diffère des autres textes dans ce corpus du fait qu'il comporte en alternance passages en prose et passages en vers. La forme de négation ne diffère pas de façon statistiquement significative selon qu'elle se trouve en vers ou en prose : pour l'ensemble des propositions (principales + subordonnées), $\chi^{2}(2, N=4)=0,73, p=.393$; pour les seules principales, test exact de Fisher, $p=.268$; pour les seules subordonnées, test exact de Fisher, $p=.599$.
} 
Registre

\begin{tabular}{lcc}
\cline { 2 - 3 } Négation & Récit & Oral représenté \\
\hline simple & $4(30,8 \%)$ & $19(45,2 \%)$ \\
renforcée & $9(69,2 \%)$ & $23(54,8 \%)$ \\
\hline \hline
\end{tabular}

Tableau 7: Aucassin et Nicolette : Négation en principale par registre

\begin{tabular}{lcc}
\hline \hline & \multicolumn{2}{c}{ Registre } \\
\cline { 2 - 3 } Négation & \multicolumn{1}{c}{ Récit } & Oral représenté \\
\hline simple & $11(84,6 \%)$ & $23(100 \%)$ \\
renforcée & $2(15,4 \%)$ & 0 \\
\hline \hline
\end{tabular}

Tableau 8: Aucassin et Nicolette : Négation en subordonnée par registre

\begin{tabular}{lcc}
\hline \hline & \multicolumn{2}{c}{ Oral représenté } \\
\cline { 2 - 3 } Négation & Hommes & Femme (Nicolette) \\
\hline simple & $18(52,9 \%)$ & $1(12,5 \%)$ \\
renforcée & $16(47,1 \%)$ & $7(87,5 \%)$ \\
\hline \hline
\end{tabular}

Tableau 9: Aucassin et Nicolette : Négation en principale - oral représenté des hommes et des femmes

Nicolette, avec sa forte préférence pour la négation renforcée, se démarque de celle des hommes, dans laquelle les taux de négation simple et renforcée se trouvent presque à égalité. Cette différence de distribution selon le sexe est presque statistiquement significative; test exact de Fisher, $p=.054$.

Dans la Queste, en revanche, l'effet du registre est tout autre : en principale, le choix entre la négation simple et renforcée varie sensiblement entre l'oral représenté (64\% renforcée) et le récit $(44,2 \%$ renforcée; tableau 10). Cette différence de distribution est statistiquement significative, $\chi^{2}(2, N=4)=10,87, p<.001$. Il n'en est pas de même pour les subordonnées (tableau 11), où la négation simple est de loin majoritaire, sans aucun effet de registre, $\chi^{2}(2, N=4)=0,22, p=.639$.

Pour ce qui est du sexe du locuteur des exemples d'oral représenté, les hommes et les femmes dans la Queste manifestent des tendances divergentes (tableau 12). En principale, alors que les hommes présentent un taux de négation renforcée de $68,8 \%$, celui des femmes ne s'élève qu'à $37 \%$. Dans ce contexte, le sexe du locuteur a un effet statistiquement significatif sur le choix de la variante négative, $\chi^{2}(2, N=4)=10,28$, $p<.05$. Les données d'oral représenté en subordonnée (tableau 13), par contre, ne révèlent aucun effet de sexe, $\chi^{2}(2, N=4)=0,07, p=.791$. 


\begin{tabular}{lcr}
\hline \hline & \multicolumn{2}{c}{ Registre } \\
\cline { 2 - 3 } Négation & Récit & Oral représenté \\
\hline simple & $53(56,1 \%)$ & $72(36 \%)$ \\
renforcée & $41(44,2 \%)$ & $128(64 \%)$ \\
\hline \hline
\end{tabular}

Tableau 10: Queste : Négation en principale par registre

\begin{tabular}{lrc}
\hline \hline & \multicolumn{2}{c}{ Registre } \\
\cline { 2 - 3 } Négation & \multicolumn{1}{c}{ Récit } & Oral représenté \\
\hline simple & $106(80,9 \%)$ & $171(78,8 \%)$ \\
renforcée & $25(19,1 \%)$ & $46(21,2 \%)$ \\
\hline \hline
\end{tabular}

Tableau 11: Queste : Négation en subordonnée par registre

\begin{tabular}{lrl}
\hline \hline & \multicolumn{2}{c}{ Oral représenté } \\
\cline { 2 - 3 } Négation & Hommes & Femmes \\
\hline simple & $53(31,2 \%)$ & $17(63,0 \%)$ \\
renforcée & $117(68,8 \%)$ & $10(37,0 \%)$ \\
\hline \hline
\end{tabular}

Tableau 12: Queste : Négation en principale - oral représenté des hommes et des femmes

L'effet du registre observé dans la Queste se reproduit dans Cassidorus, où l'on constate, encore une fois, que le taux de négation renforcée en principale varie de manière significative selon le registre (tableau 14). Tout comme dans la Queste, l'oral représenté se trouve nettement en avance, par rapport au récit, en adoptant la variante renforcée; l'effet du registre est statistiquement significatif pour les principales, $\chi^{2}(2, N=4)=15,09, p<.0001$, mais il disparaît en subordonnée (tableau 15), $\chi^{2}(2, N=4)=0,22, p=.639$.

Si les hommes ont plus tendance que les femmes à employer la négation renforcée en principale (tableau 16), comme dans la Queste, la différence de distribution n'en atteint pas toutefois la valeur statistiquement significative, $\chi^{2}(2, N=4)=1,86$, $p=.173$. De même, aucun effet de sexe n'est visible en subordonnée (tableau 17), test exact de Fisher, $p=.182$.

Chez Joinville, à la différence de la Queste et de Cassidorus, le registre n'influe pas sur la distribution des variantes négatives, $\chi^{2}(2, N=4)=.01, p=.92$. Comme on le voit dans le tableau 18, la négation renforcée demeure plus fréquente que la négation simple en principale, sans que cette distribution varie selon le registre. 


\begin{tabular}{lrr}
\hline \hline & \multicolumn{2}{c}{ Oral représenté } \\
\cline { 2 - 3 } Négation & Hommes & Femmes \\
\hline simple & $133(78,2 \%)$ & $36(80,0 \%)$ \\
renforcée & $37(21,8 \%)$ & $9(20,0 \%)$ \\
\hline \hline
\end{tabular}

Tableau 13: Queste : Négation en subordonnée - oral représenté des hommes et des femmes

\begin{tabular}{lrr}
\hline \hline & \multicolumn{2}{c}{ Registre } \\
\cline { 2 - 3 } Négation & \multicolumn{1}{c}{ Récit } & Oral représenté \\
\hline simple & $151(77,0 \%)$ & $100(58,1 \%)$ \\
renforcée & $45(23,0 \%)$ & $72(41,9 \%)$ \\
\hline \hline
\end{tabular}

Tableau 14: Cassidorus : Négation en principale par registre

\begin{tabular}{|c|c|c|}
\hline \multirow[b]{2}{*}{ Négation } & \multicolumn{2}{|c|}{ Registre } \\
\hline & Récit & Oral représenté \\
\hline simple & $160(88,4 \%)$ & $135(90,0 \%)$ \\
\hline renforcée & $21(11,6 \%)$ & $15(10,0 \%)$ \\
\hline
\end{tabular}

Tableau 15: Cassidorus : Négation en subordonnée par registre

\begin{tabular}{lcc}
\hline \hline & \multicolumn{2}{c}{ Oral Représenté } \\
\cline { 2 - 3 } Négation & Hommes & Femmes $^{1}$ \\
\hline simple & $69(56,6 \%)$ & $23(69,7 \%)$ \\
renforcée & $53(43,4 \%)$ & $10(30,3 \%)$ \\
\hline \hline
\end{tabular}

${ }^{1}$ Ces données excluent celles d'Helcanor, femme déguisée en homme pendant la plupart du récit.

Tableau 16: Cassidorus : Négation en principale - oral représenté des hommes et des femmes

Tout comme dans les textes précédents, les subordonnées ne contiennent qu'un taux assez faible de négation renforcée (tableau 19), sans différenciation significative selon le registre, test exact de Fisher, $p=.299$.

L'absence de négation phrastique dans l'oral représenté des femmes empêche d'investiguer l'effet du sexe dans Joinville. 


\begin{tabular}{lcr}
\hline \hline & \multicolumn{2}{c}{ Oral représenté } \\
\cline { 2 - 3 } Négation & Hommes & Femmes ${ }^{1}$ \\
\hline simple & $84(92,3 \%)$ & $28(84,8 \%)$ \\
renforcée & $7(7,7 \%)$ & $5(15,2 \%)$ \\
\hline \hline
\end{tabular}

${ }^{1}$ Ces données excluent celles d'Helcanor, femme déguisée en homme pendant la plupart du récit.

Tableau 17: Cassidorus : Négation en subordonnée - oral représenté des hommes et des femmes

\begin{tabular}{lcc}
\hline \hline & \multicolumn{2}{c}{ Registre } \\
\cline { 2 - 3 } Négation & Récit & Oral représenté \\
\hline simple & $21(43,8 \%)$ & $12(42,9 \%)$ \\
renforcée & $27(56,3 \%)$ & $16(57,1 \%)$ \\
\hline \hline
\end{tabular}

Tableau 18: Joinville : Négation en principale par registre

\begin{tabular}{lrr}
\hline \hline & \multicolumn{2}{c}{ Registre } \\
\cline { 2 - 3 } Négation & \multicolumn{1}{c}{ Récit } & Oral représenté \\
\hline simple & $116(81,7 \%)$ & $23(88,5 \%)$ \\
renforcée & $26(18,3 \%)$ & $3(11,5 \%)$ \\
\hline \hline
\end{tabular}

Tableau 19: Joinville : Négation en subordonnée par registre

Dans notre dernier texte, Saintré, le registre ne semble avoir aucune incidence sur le choix de variante négative, ni en principale (tableau $20 ; \chi^{2}(2, N=4)=.61$, $p=.435)$, ni en subordonnée (tableau $\left.21 ; \chi^{2}(2, N=4)=.35, p=.554\right)$.

De même, les données d'oral représenté ne révèlent aucun effet de sexe, ni en principale (tableau 22; $\chi^{2}(2, N=4)=1,06, p=.303$ ), ni en subordonnée (tableau $\left.23 ; \chi^{2}(2, N=4)=1,95, p=.163\right)$.

\section{DISCUSSION}

En résumé, les résultats principaux sont les suivants. De prime abord, il existe dans chaque texte une asymétrie entre principales et subordonnées (tableaux 5 et 6), en ce sens que la négation renforcée est toujours plus fréquente en principale; c'est déjà la variante majoritaire dans trois des textes, alors qu'en subordonnée, elle représente tout au plus $20 \%$ des négations phrastiques dans ce corpus. Ces résultats corroborent 


\begin{tabular}{lcc}
\hline \hline & \multicolumn{2}{c}{ Registre } \\
\cline { 2 - 3 } Négation & Récit & Oral représenté \\
\hline simple & $58(65,2 \%)$ & $70(59,8 \%)$ \\
renforcée & $31(34,8 \%)$ & $47(40,2 \%)$ \\
\hline \hline
\end{tabular}

Tableau 20: Saintré : Négation en principale par registre

\begin{tabular}{lrc}
\hline \hline & \multicolumn{2}{c}{ Registre } \\
\cline { 2 - 3 } Négation & Récit & Oral représenté \\
\hline simple & $111(80,4 \%)$ & $89(77,4 \%)$ \\
renforcée & $27(19,6 \%)$ & $26(22,6 \%)$ \\
\hline \hline
\end{tabular}

Tableau 21: Saintré : Négation en subordonnée par registre

\begin{tabular}{lcc}
\hline \hline \multirow{2}{*}{ Négation } & \multicolumn{2}{c}{ Oral représenté } \\
\cline { 2 - 3 } & Hommes & Femmes \\
\hline simple & $33(60,0 \%)$ & $25(50,0 \%)$ \\
renforcée & $22(40,0 \%)$ & $25(50,0 \%)$ \\
\hline \hline
\end{tabular}

Tableau 22: Saintré : Négation en principale - oral représenté des hommes et des femmes

\begin{tabular}{lrr}
\hline \hline & \multicolumn{2}{c}{ Oral représenté } \\
\cline { 2 - 3 } Négation & Hommes & Femmes \\
\hline simple & $42(84,0 \%)$ & $46(73,0 \%)$ \\
renforcée & $8(16,0 \%)$ & $17(27,0 \%)$ \\
\hline \hline
\end{tabular}

Tableau 23: Saintré : Négation en subordonnée - oral représenté des hommes et des femmes

ceux qu'ont obtenus Schøsler et Völker (2014) pour d'autres textes et semblent appuyer l'observation que les subordonnées représentent un contexte linguistique conservateur en comparaison avec les principales (Givón 1976, Hock 1991). En français, la négation renforcée se serait donc diffusée à partir des principales, pour toucher ensuite les subordonnées. Dans le présent corpus, les proportions de négation renforcée en principale ne révèlent pas clairement une évolution 
diachronique, même si une telle évolution devait y être présente. Dans les subordonnées du corpus, par contre, les proportions de négation renforcée vont globalement croissant; il semble qu'avec ces textes, l'on n'est que peu éloigné du moment où la négation renforcée a atteint les subordonnées, alors que sa présence en principale date de plus longtemps. La présente analyse adopte la perspective de Kroch (1989), qui voit dans le changement diachronique la présence de plusieurs grammaires en compétition. En principale, la variante novatrice se trouve bien implantée et rivalise fortement avec la variante conservatrice. Par contre, si la variante novatrice commence à faire des percées en subordonnée, elle reste néanmoins nettement minoritaire dans ce contexte.

Dans les présentes données, la distribution des variantes conservatrice (ne seul) et novatrice (ne ...pas/mie) en principale s'avère parfois conditionnée par des facteurs sociostylistiques. En effet, quelques-uns de nos textes (Queste, Cassidorus) révèlent une différence statistiquement significative entre la distribution de la négation simple et renforcée en oral représenté et en récit; dans ces cas, c'est toujours l'oral représenté qui est en avance sur le récit en adoptant la forme novatrice (négation renforcée). Ces résultats, compatibles avec ceux de Romaine (1982), Donaldson (2014), Glikman et Mazziotta (2014), et Steiner (2014), révèlent un effet de registre et suggèrent que les auteurs des textes, du moins certains d'entre eux, variaient systématiquement leurs choix de négation à des buts stylistiques. ${ }^{3}$

L'hypothèse de Kroch veut que, lors d'un changement en cours, chaque locuteur dispose de plusieurs grammaires mentales qui coexistent, et que ces différentes grammaires - toujours chez un même individu - représentent chacune une étape distincte du changement. Si tel est le cas, on s'attend précisément à la différenciation de formes conservatrices et novatrices selon le registre observée dans la Queste et dans Cassidorus. En effet, chacun de ces textes contient non pas une seule grammaire, mais de multiples grammaires en compétition, dont chacune représente une étape du changement en cours. Du moins pour la grammaire de la négation, ce serait une erreur de croire à l'homogénéité de chaque texte. Étant donné le sens de la variation (davantage de formes renforcées/novatrices en oral représenté), on est à même de soutenir l'hypothèse que la négation renforcée en ancien français représente un changement d'en bas, qui s'emploie d'abord dans les registres informels et populaires avant d'atteindre peu à peu les registres plus soutenus.

Que l'oral représenté soit différencié également selon le sexe dans la Queste corrobore les résultats de Donaldson (2014) sur un autre changement en cours dans ce même texte. Pour ce qui est de la négation, tout comme pour la croissance des principales en SV, les femmes dans la Queste présentent un comportement plus conservateur que celui des hommes : elles ont davantage tendance à employer la variante ancienne. Dans un article précédent (Donaldson 2014), j'ai avancé l'hypothèse que les femmes, ayant le plus souvent des rôles familial et ménager dans la société médiévale française (voir Power 1975), figuraient dans des réseaux sociaux denses (Milroy et Milroy 1985), qui favorisent peu la diffusion d'une innovation.

\footnotetext{
${ }^{3}$ Rappelons cependant que Schøsler et Völker (2014) n'ont trouvé aucun effet du registre dans leurs données.
} 
Inversement, les hommes, dont les rôles sociaux dépassaient typiquement le foyer et entraînaient des contacts avec un grand nombre de locuteurs divers, participaient à des réseaux sociaux lâches, susceptibles de faciliter la diffusion d'innovations linguistiques. Les résultats obtenus pour la Queste, qui reposent sur un assez grand nombre d'exemples, soutiennent cette hypothèse. Le cas de Nicolette dans Aucassin et Nicolette, en revanche, est moins clair; comme le montre le tableau 9, l'oral représenté de Nicolette marque une préférence presque catégorique pour la négation renforcée en principale, à la différence des hommes dans ce texte. Signalons tout d'abord la faible quantité d'exemples, qui, d'emblée, rend hasardeuse quelle qu'interprétation que ce soit. Mais pour me livrer à la spéculation, le comportement novateur de Nicolette serait-il un tour de l'auteur pour mettre en relief le fait qu'elle est étrangère, d'une société tout autre que celle d'Aucassin et des siens? Ou bien une façon de laisser entrevoir que les réseaux sociaux auxquels participent Nicolette seraient plus lâches - Nicolette est après tout une femme noble et jouit d'un statut social bien au-dessus de celui de la plupart des femmes dans la société médiévale - et par conséquent dissemblables de ceux des femmes typiques de l'époque? Nicolette étant le seul personnage féminin important de l'œuvre, de telles possibilités restent nécessairement au stade de la spéculation.

La distribution des formes simple et renforcée selon le registre n'apparaît pas dans tous les textes du corpus modeste constitué ici. En effet, dans Aucassin et Nicolette, chez Joinville et dans Saintré, le taux des deux variantes en compétition ne varie pas de façon significative. Pourtant, ces textes (surtout Joinville et Saintré) datent d'une époque où, selon les diachroniciens, la négation se trouvait en pleine évolution. Ceci implique un certain arbitraire en ce que certains textes reflètent l'évolution de la négation en manifestant de la variation intratextuelle, alors que d'autres ne le font pas. Chez Joinville, par exemple, je n'ai pu mettre au jour des traces de variation de registre ni pour l'évolution vers la grammaire SV (Donaldson 2014) ni pour le développement de négation renforcée, alors que la Queste témoigne de ce genre de variation dans les deux cas de figure. Il serait donc peu réaliste de s'attendre à ce que chaque texte se comporte de façon comparable sur ce point; dans le même esprit, van Reenen et Schøsler (1995) ont démontré que le marquage de la structure informationnelle dépend du genre de texte. Il se peut que Joinville et Saintré soient des textes conservateurs (voir à ce sujet Balon et Larrivée 2016 pour Saintré), ou bien que les auteurs se soient trouvés à l'abri de ces changements, ou enfin que, pour des raisons que nous ignorons pour l'instant, le changement ne leur était pas très perceptible. En tout cas, pour la négation, ils n'ont pas varié leur style, ce qui n'exclut pas la possibilité que d'autres facteurs sociopragmatiques, telle la structure informationnelle, influent sur la distribution des variantes simple et renforcée dans ces textes.

Les présents résultats plaident en faveur d'un étiquetage de corpus qui tient compte des détails sociostylistiques telle la distinction entre oral représenté et récit. Les données de la BFM et du corpus MCVF bénéficient déjà de cette annotation. Le sexe du locuteur, par contre, qui influe sur la variation dans au moins quelques textes historiques examinés sous cet angle jusqu'à présent, n'est encore marqué dans aucun corpus d'ancien français, à ma connaissance. L'approche variationniste 
à la Labov (1972) requiert un très grand nombre de données, surtout lorsque l'analyse met en jeu un nombre important de facteurs. Cela va sans dire que l'intérêt des corpus annotés consiste à fournir aux chercheurs de nombreux renseignements détaillés sur autant d'exemples que possible. Une fois disponibles dans des corpus consultables, les détails sociostylistiques seront d'une aide précieuse pour les chercheurs qui ont pour objectifs de mieux cerner le rôle de différents facteurs sociaux dans la diffusion d'un changement et d'approfondir la description sociosituationnelle des langues anciennes.

\section{CONCLuSION}

Se situant dans le cadre des études de linguistique sociohistorique, cet article se veut une modeste contribution à la connaissance sociolinguistique d'un changement diachronique bien connu et souvent discuté en linguistique historique française : l'évolution d'une négation phrastique simple en ne vers la forme double du type ne...pas. En opposant deux registres distincts au sein d'un même texte, les résultats révèlent des exemples de variation intratextuelle; dans certains textes du corpus, la négation en oral représenté présente un taux de négation renforcée (la variante novatrice dans le changement) supérieur à celui du récit. De même, dans un des textes, les hommes, vus par le biais de leurs exemples d'oral représenté, font preuve d'un comportement linguistique plus novateur que les femmes. Quoiqu'il s'agisse de premiers résultats provenant d'un échantillon de textes limité, les observations viennent corroborer celles de plusieurs études antérieures (Donaldson 2014, Glikman et Mazziotta 2014, Steiner 2014) et légitiment une enquête semblable sur une plus grande échelle. Pris ensemble, ces résultats ajoutent des détails sociohistoriques, jusque-là inaperçus, à la compréhension de la diffusion de ce changement diachronique, qui, malgré bon nombre d'études, reste incomplète. Les résultats soulignent en même temps l'importance d'un étiquetage de corpus comprenant des détails d'ordre sociostylistique telle la distinction entre récit et oral représenté, et pour ce dernier, le sexe du "locuteur."

\section{GEUVRES LITTÉRAIRES CITÉES}

Aucassin et Nicolette. Éd. J. Dufournet, 1984. Paris : Flammarion. de la Sale, Antoine. Jehan de Saintré. Éd. J. Blanchard, 1995. Paris : Librairie Générale Française.

de Joinville, Jehan. La vie de Saint Louis. Éd. N. Corbett, 1977. Sherbrooke : Naaman. Queste del saint graal. Éd. C. Marchello-Nizia, 2011. <http://portal.textometrie.org/txm/> Roman de Cassidorus. Éd. J. Palermo, 1963. Paris : Société des anciens textes français.

\section{RÉFÉRENCES}

Ayres-Bennett, Wendy. 2001. Socio-historical linguistics and the history of French. Journal of French Language Studies 11(2) : 159-177. 
Ayres-Bennett, Wendy. 2004. Sociolinguistic variation in seventeenth-century French. Cambridge : Cambridge University Press.

Balon, Laurent, et Pierre Larrivée. 2016. L'ancien français n'est déjà plus une langue à sujet nul : nouveau témoignage des textes légaux. Journal of French Language Studies 26(2): 221-237.

BFM - Base de Français Médiéval. 2012. Lyon : ENS de Lyon, Laboratoire ICAR. <http://bfm. ens-lyon.fr>

Buridant, Claude. 2000. Grammaire nouvelle de l'ancien français. Paris : Sedes.

Donaldson, Bryan. 2014. Socio-stylistic reflexes of syntactic change in Old French. Journal of French Language Studies 24(3) : 319-345.

Einhorn, E. 1974. Old French : A concise handbook. Cambridge : Cambridge University Press.

Fleischman, Suzanne. 1990. Tense and narrativity : From medieval performance to modern fiction. Austin : University of Texas Press.

Foulet, Lucien. 1974. Petite syntaxe de l'ancien français. Paris : Honoré Champion. [1919].

Givón, Talmy. 1976. Topic, pronoun, and grammatical agreement. Dans Subject and topic, sous la direction de Charles N. Li, 149-188. New York : Academic Press.

Glikman, Julie et Nicolas Mazziotta. 2014. Représentation de l'oral et syntaxe dans la prose de la Queste del saint graal (1225-1230). Dans Représentation du sens linguistique 5, sous la direction de Dominique Lagorgette et Pierre Larrivée, 43-64. Chambéry : Éditions de l'Université de Savoie.

Guiraud, Pierre. 1964. L'opposition actuel-virtuel : remarques sur l'adverbe de négation dans "Aucassin et Nicolette". Dans Mélanges de linguistique romane et de philologie médiévale offerts à M. Maurice Delbouille, sous la direction de Jean Renson, 295-306. Gembloux : Duculot.

Harris, Martin. 1988. French. Dans The Romance languages, sous la direction de Martin Harris et Nigel Vincent, 209-245. Oxford : Oxford University Press.

Hock, Hans H. 1991. Principles of historical linguistics. $2^{\mathrm{e}}$ édition. Berlin : De Gruyter.

Koch, Peter et Wulf Oesterreicher. 1985. Sprache der Nähe - Sprache der Distanz : Mündlichkeit und Schriftlichkeit im Spannungsfeld von Sprachtheorie und Sprachgeschichte [Langage de proximité - langage de distance : oralité et scripturalité au croisement de la théorie du langage et de la linguistique historique]. Romanistisches Jahrbuch (36) : 15-43.

Kroch, Anthony. 1989. Reflexes of grammar in patterns of language change. Language Variation and Change 1(3) : 199-244.

Labov, William. 1972. Sociolinguistic patterns. Philadelphia : University of Pennsylvania Press.

Labov, William. 1994. Principles of linguistic change : Internal factors. Oxford : Blackwell.

Larrivée, Pierre. 2010. The pragmatic motifs of the Jespersen cycle : Default, activation, and the history of negation in French. Lingua 120(9) : 2240-2258.

Larrivée, Pierre. 2011. Is there a Jespersen cycle? Dans The evolution of negation: Beyond the Jespersen cycle, sous la direction de Pierre Larrivée et Richard Ingham, 1-22. Berlin : Walter de Gruyter.

Larrivée, Pierre et Richard Ingham, éd. 2011. The evolution of negation : Beyond the Jespersen cycle. Berlin : DeGruyter Mouton.

Lodge, R. Anthony. 2004. A sociolinguistic history of Parisian French. Cambridge : Cambridge University Press.

Marchello-Nizia, Christiane. 2003. Le français dans l'histoire. Dans Le grand livre de la langue française, sous la direction de Marina Yaguello, 11-90. Paris : Seuil.

Marchello-Nizia, Christiane. 2012. L'oral représenté en français médiéval, un accès construit à une face cachée des langues mortes. Dans Le changement en français : études de 
linguistique diachronique, sous la direction de Céline Guillot, Bernard Combettes, Alexei Lavrentiev, Evelyne Oppermann-Marsaux et Sophie Prévost, 247-264. Berne : Peter Lang.

Martineau, France. 2005. Perspectives sur le changement linguistique : aux sources du français canadien. Canadian Journal of Linguistics 50(1-4) : 173-213.

Martineau, France. 2009. Modeling change : A historical sociolinguistics perspective on French negation. Dans Corpus analysis and variation in linguistics, sous la direction de Yuji Kawaguchi, Makoto Minegishi et Jacques Durand, 159-178. Amsterdam : John Benjamins.

Martineau, France. 2011. Ne-absence in declarative and yes/no interrogative contexts : Some patterns of change. Dans The evolution of negation : Beyond the Jespersen cycle, sous la direction de Pierre Larrivée et Richard Ingham, 179-207. Berlin : Walter de Gruyter.

Martineau, France, Paul Hirschbühler, Anthony Kroch et Yves Charles Morin. 2010. Corpus MCVF annoté syntaxiquement. Modéliser le changement : les Voies du français. Université d'Ottawa : Département de Français. <http://www.voies.uottawa.ca/projet_fr. html>

Martineau, France et Raymond Mougeon. 2003. A sociolinguistic study of the origins of ne deletion in European and Quebec French. Language 79(1) : 118-152.

Milroy, James et Lesley Milroy. 1985. Linguistic change, social network and speaker innovation. Journal of Linguistics 21(2) : 339-384.

Mosegaard Hansen, Maj-Britt. 2009a. Forms of sentence negation in a 14th-century French text : A cognitive/functional analysis. Quaderns de Filologia. Estudis lingüistics 14 : $153-168$.

Mosegaard Hansen, Maj-Britt. 2009b. The grammaticalization of negative reinforcers in Old and Middle French : A discourse-functional approach. Dans Current trends in diachronic semantics and pragmatics, sous la direction de Maj-Britt Mosegaard Hansen et Jacqueline Visconti, 227-251. Bingley, UK : Emerald.

Mosegaard Hansen, Maj-Britt. 2011. Negative cycles and grammaticalization. Dans The Oxford handbook of grammaticalization, sous la direction de Heiko Narrog et Bernd Heine, 570-579. Oxford : Oxford University Press.

Mosegaard Hansen, Maj-Britt et Jacqueline Visconti. 2009. On the diachrony of "reinforced" negation in French and Italian. Dans Grammaticalization and pragmatics : Facts, approaches, theoretical issues, sous la direction de Corinne Rossari, Claudia Ricci et Adriana Spiridon, 137-171. Bingley, UK : Emerald.

Mosegaard Hansen, Maj-Britt. 2014. The grammaticalization of negative indefinites: The case of the temporal/aspectual n-words plus and mais in Medieval French. Dans The diachrony of negation, sous la direction de Maj-Britt Mosegaard Hansen et Jacqueline Visconti, 185-212. Amsterdam : John Benjamins.

Mosegaard Hansen, Maj-Britt et Jacqueline Visconti, dirs. 2014. The diachrony of negation. Amsterdam : John Benjamins.

Nevalainen, Terttu et Helena Raumolin-Brunberg. 2003. Historical sociolinguistics. London : Longman.

O'Connor, B. F. 1881. The negative particle "mie" in Old French. American Journal of Philology 2(6) : 210-212.

Posner, Rebecca. 1996. The Romance languages. Cambridge : Cambridge University Press.

Posner, Rebecca. 1997. Linguistic change in French. Oxford : Oxford University Press.

Power, Eileen. 1975. Medieval women. Cambridge : Cambridge University Press.

Price, Glanville. 1962. The negative particles pas, mie and point in French. Linguisticum 14 : 14-34. 
Price, Glanville. 1971. The French language : Present and past. London : Edward Arnold. Price, Glanville. 1997. Negative particles in French. Dans De mot en mot : Aspects of medieval linguistics, sous la direction de Stewart Gregory et David A. Trotter, 173-190. Cardiff : University of Wales Press.

van Reenen, Pieter et Lene Schøsler. 1995. The thematic structure of the main clause in Old French : Or versus si. Dans Historical Linguistics 1993 : Selected papers from the 11th International Conference on Historical Linguistics, sous la direction de Henning Andersen, 401-419. Amsterdam : John Benjamins.

Rodríguez Somolinos, Amalia. 2013. Présentation. Dans Diachroniques 3 : Marques d'oralité en français médiéval, sous la direction de Amalia Rodríguez Somolinos.

Romaine, Suzanne. 1982. Socio-historical linguistics. Cambridge : Cambridge University Press.

Schøsler, Lene et Harald Völker. 2014. Intralinguistic and extralinguistic variation factors in Old French negation with ne- $\emptyset$, ne-mie, ne-pas and ne-point across different text types. Journal of French Langauge Studies 24(1) : 127-153.

Steiner, B. Devan. 2014. The evolution of information structure and verb second in the history of French. Thèse de doctorat, Indiana University, Bloomington.

Vance, Barbara. 1981. A syntactic and semantic study of subject personal pronoun usage in Old French. Mémoire de maîtrise, Cornell University.

Vance, Barbara. 1997. Syntactic change in medieval French : Verb-second and null subjects. Dordrecht : Kluwer.

Vance, Barbara, Bryan Donaldson et B. Devan Steiner. 2010. V2 loss in Old French and Old Occitan : The role of fronted clauses. Dans Romance linguistics 2009 : Selected papers from the 39th Linguistic Symposium on Romance Languages, sous la direction de Sonia Colina, Antxon Olarrea et Ana Maria Carvalho, 301-320. Amsterdam : John Benjamins.

Vennemann, Theo. 1974. Topics, subjects, and word order : From SXV to SVX via TVX. Dans Historical linguistics I : Syntax, morphology, internal and comparative reconstruction, sous la direction de John M. Anderson et Charles Jones, 339-376. Amsterdam : North Holland.

Völker, Harald. 2007. A 'practice of the variant' and the origins of the standard : Presentation of a variationist linguistics method for a corpus of Old French charters. Journal of French Language Studies 17(2) : 207-223.

van Wartburg, Walter. 1971. Évolution et structure de la langue française. 10e édition. Berne : A. Francke. 